$2-1-2008$

\title{
Atomic Size Mismatch Strain Induced Surface Reconstructions
}

Jessica E. Bickel

Cleveland State University, j.e.bickel@csuohio.edu

Normand A. Modine

Sandia National Laboratories

Anton Van der ven

University of Michigan

Joanna Mirecki Millunchick

University of Michigan

Follow this and additional works at: https://engagedscholarship.csuohio.edu/sciphysics_facpub

Part of the Physics Commons

How does access to this work benefit you? Let us know!

Publisher's Statement

(C) 2008 American Institute of Physics

\section{Repository Citation}

Bickel, Jessica E.; Modine, Normand A.; Van der ven, Anton; and Millunchick, Joanna Mirecki, "Atomic Size Mismatch Strain Induced Surface Reconstructions" (2008). Physics Faculty Publications. 191.

https://engagedscholarship.csuohio.edu/sciphysics_facpub/191

This Article is brought to you for free and open access by the Physics Department at EngagedScholarship@CSU. It has been accepted for inclusion in Physics Faculty Publications by an authorized administrator of

EngagedScholarship@CSU. For more information, please contact library.es@csuohio.edu. 


\section{Atomic size mismatch strain induced surface reconstructions}

Jessica E. Bickel, Normand A. Modine, Anton Van der Ven, and Joanna Mirecki Millunchick'

Citation: Appl. Phys. Lett. 92, 062104 (2008); doi: 10.1063/1.2841846

View online: http://dx.doi.org/10.1063/1.2841846

View Table of Contents: http://aip.scitation.org/toc/apl/92/6

Published by the American Institute of Physics 


\title{
Atomic size mismatch strain induced surface reconstructions
}

\author{
Jessica E. Bickel, ${ }^{1}$ Normand A. Modine,${ }^{2}$ Anton Van der Ven, ${ }^{1}$ and \\ Joanna Mirecki Millunchick ${ }^{1, a)}$ \\ ${ }^{1}$ Department of Materials Science and Engineering, University of Michigan, Ann Arbor, Michigan 48109, \\ USA \\ ${ }^{2}$ Sandia National Laboratories, Albuquerque, New Mexico 87185, USA
}

(Received 9 November 2007; accepted 21 January 2008; published online 13 February 2008)

\begin{abstract}
The effects of lattice mismatch strain and atomic size mismatch strain on surface reconstructions are analyzed using density functional theory. These calculations demonstrate the importance of an explicit treatment of alloying when calculating the energies of alloyed surface reconstructions. Lattice mismatch strain has little impact on surface dimer ordering for the $\alpha 2(2 \times 4)$ reconstruction of GaAs alloyed with In. However, atomic size mismatch strain induces the surface In atoms to preferentially alternate position, which, in turn, induces an alternating configuration of the surface anion dimers. These results agree well with experimental data for $\alpha 2(2 \times 4)$ domains in InGaAs/GaAs surfaces. (C) 2008 American Institute of Physics. [DOI: 10.1063/1.2841846]
\end{abstract}

Many material systems exhibit surface reconstructions, where surface atoms rearrange from their bulk positions to reduce the number of dangling bonds and thereby lower the surface energy. The resulting surface reconstructions have a significant effect on epitaxial film growth ${ }^{1}$ and self-assembly at the nano- and micro-scale. ${ }^{2}$ Thus, understanding surface reconstructions and developing the ability to select a particular structure is of great value.

In pure semiconductor systems, the surface reconstructions are reasonably well understood. Surface reconstructions for $\mathrm{GaAs}^{3}$ and $\mathrm{InAs}^{4}$ have been determined experimentally through the use of reflection high energy electron diffraction and in situ scanning tunneling microscopy (STM). Ab initio calculations based on density functional theory (DFT) capture the principal factors influencing reconstruction stability in these systems and have reproduced the experimentally observed sequence of reconstructions with varying chemical potential for the $\mathrm{GaAs}^{5}$ and $\mathrm{InAs}^{4}$ (001) surfaces.

The surface reconstructions of alloyed semiconductors are more complicated, as the presence of multiple components introduces additional factors that can influence the surface structure, including lattice mismatch strain, the strain associated with the difference between the average lattice parameter of the film and substrate, and atomic size mismatch strain, the strain associated with the bond length differences of the alloy constituents. The effects of these two strains are very difficult to distinguish experimentally as they cannot be independently varied. Recent work shows that lattice mismatch strain influences surface reconstruction in semiconductor films by shifting the relative surface energies of different reconstructions. DFT calculations of InAs show shifts in reconstruction stability as a function of applied strain. ${ }^{6}$ Lattice mismatch strain has also been shown to change surface reconstructions for Ge films grown on $\mathrm{Sb}$ covered $\mathrm{Si}(111)$ substrates, with the reconstruction changing as a function of strain induced by film thickness. ${ }^{7}$ The work presented in this letter shows that atomic size mismatch strain due to surface segregated alloying elements also plays an important role in affecting the relative stability of competitive surface reconstructions.

\footnotetext{
${ }^{a)}$ Electronic mail: joannamm@umich.edu.
}

In this paper, we examine the role of homogeneous lattice mismatch strain and localized atomic mismatch strain on the structure of surface reconstructions, focusing on the stability of different As dimer arrangements in the $\alpha 2(2 \times 4)$ reconstruction for GaAs alloyed with In. DFT calculations demonstrate that atomic size mismatch strain resulting from the $7 \%$ difference in equilibrium bond length of InAs and GaAs imposes ordering of the In atoms in the subsurface layer. This subsurface ordering, in turn, imposes an alternating dimer surface configuration, demonstrating the importance of explicitly including alloying in surface energy calculations. These results have been compared to experimental results in $\mathrm{In}_{0.27} \mathrm{Ga}_{0.73} \mathrm{As} / \mathrm{GaAs}$ films which exhibit small domains of the $\alpha 2(2 \times 4)$ reconstruction having a prevalence of the alternating surface dimer configuration.

The $\alpha 2(2 \times 4)$ reconstruction is common to many pure III-As semiconductors including GaAs and InAs. ${ }^{4}$ It consists of a row of As surface dimers along the [1] 0$]$ direction occupying one of two positions in the unit cell as shown in the atomistic models of Figs. 1(a) and 1(b). Since the $\alpha 2(2 \times 4)$ reconstruction requires only half occupancy of the surface dimer sites, many possible dimer arrangements are possible, including an ordered straight row, a zigzag ordering, and a disordered state with dimers stochastically distributed over half the dimer sites, as is typically observed for homoepitaxial films. ${ }^{4}$ Statistical analysis of published images of pure InAs $s^{4,8,9}$ shows the incidence of alternating dimers to be between $50 \%$ and $65 \%$, consistent with a nearly random dimer configuration.

It is unknown what effect alloying may have on dimer ordering in the $\alpha 2(2 \times 4)$ reconstruction. In order to investigate the relative importance of lattice mismatch and atomic size mismatch strain on the dimer configuration of the $\alpha 2(2 \times 4)$, we have studied the two atomistic models shown in Figs. 1(a) and 1(b). Figure 1(b) is the $R$ model, consisting of a straight row of dimers along the [1]0], and Fig. 1(a) is the $Z$ model, consisting of a row of alternating dimers along the $[1 \overline{1} 0]$. Energy calculations were performed using the Vienna $a b$ initio simulation package ${ }^{10}$ (VASP) using ultrasoft pseudopotentials $^{10,11}$ and the local density approximation (LDA). ${ }^{12}$ Both models were studied using slabs consisting of 


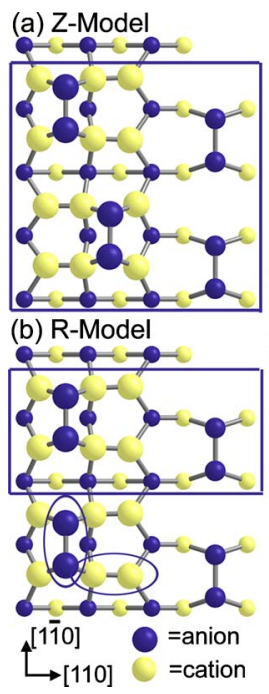

(c)

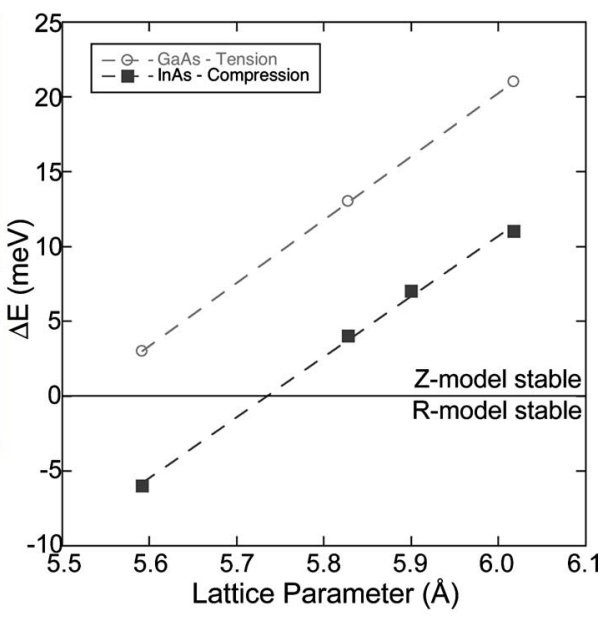

FIG. 1. (Color online) Schematic of (a) $Z$ model and (b) $R$ model configurations. DFT unit cells are outlined with rectangles and circles outline one anion surface dimer, and one cation-cation dimer. (c) The energy difference of the $R$ model and $Z$ model configurations as a function of lattice parameter for GaAs (open circles) and InAs (closed squares) slabs. $\Delta E$ $=\operatorname{energy}(R$ model $)-\operatorname{energy}(Z$ model $)$; therefore, a positive energy means the $Z$ model is more stable.

four bilayers of bulk III-V material terminated on the top by the model and on the bottom by pseudohydrogens which charge neutralize the surface. Slabs were separated by $14 \AA$ of vacuum to minimize interactions. Both the pseudohydrogen and the bottom bilayer of III-V material were kept fixed to imitate a bulk periodic crystal while the rest of the slab was allowed to relax. $6 \times 3$ and $3 \times 3 k$-point meshes were used for the $(2 \times 4)$ and $(4 \times 4)$ slabs, respectively. The plane-wave energy cutoff was set at $203.1 \mathrm{eV}$ for all calculations, the electronic temperature $(k T)$ was set to $0.025 \mathrm{eV}$, and slabs were relaxed with a VASP relaxation tolerance of $0.1 \mathrm{meV}$. All energies are reported per $(2 \times 4)$ unit cell.

Figure 1(c) shows the energy difference between the $R$ model and the $Z$ model for pure GaAs and InAs slabs, at LDA lattice parameters ranging from GaAs (5.592 $\AA$ ) to InAs $(6.018 \AA)$. A positive number in Fig. 1(c) indicates that the $Z$ model is more stable than the $R$ model. For pure, relaxed GaAs at the GaAs lattice parameter, the $Z$ model is stabilized relative to the $R$ model by $3 \mathrm{meV}$ per $(2 \times 4)$ unit cell. However, tensile strain enhances this stability to $21 \mathrm{meV}$ for pure GaAs at the InAs lattice parameter. For pure, relaxed InAs the $Z$ model is again predicted to be stable, in this case by $11 \mathrm{meV}$. This relative stability decreases as the slab is subjected to a compressive strain until the lattice parameter reaches $5.74 \AA$, below which the $R$ model is stabilized.

While the $Z$ model is predicted to be stable for both pure GaAs and InAs, it is only marginally stable relative to the $R$ model, suggesting that thermal fluctuations can easily disorder the alternating dimer arrangement of the $Z$ model at finite temperature. Monte Carlo simulations were applied to a lattice-model Hamiltonian describing the configurational energy of As dimers on the $\alpha 2(2 \times 4)$ reconstruction. Simulations for pure InAs at the InAs lattice parameter show that the incidence of the As dimers alternating position on the surface is $53 \%$ at typical growth conditions of $T=475{ }^{\circ} \mathrm{C}$, A I G H TS L I I K K (1) only $61 \%$ at $T=0{ }^{\circ} \mathrm{C},{ }^{13}$ which correlates

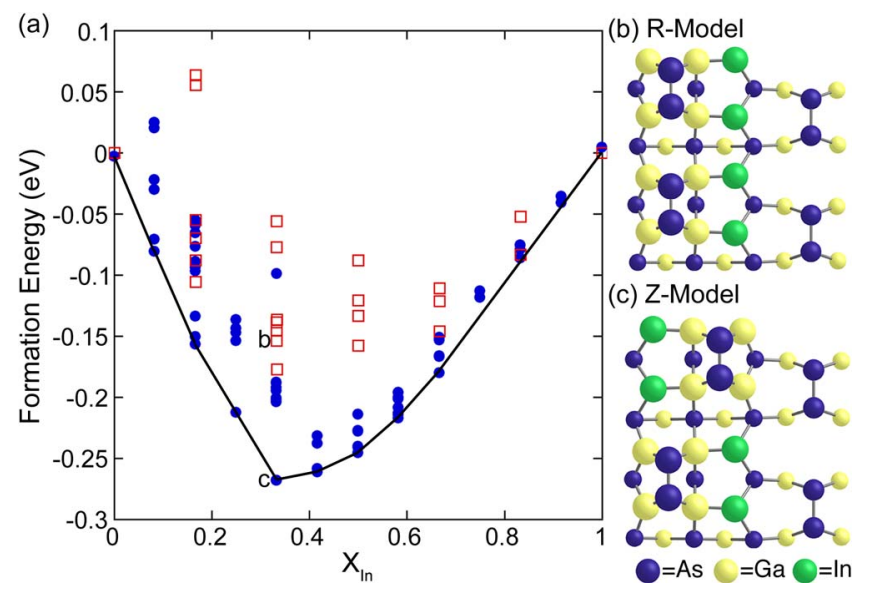

FIG. 2. (Color online) Convex hull of the formation energy of different configurations of In in the $Z$ model (closed circles) and $R$ model (open squares) with schematics of configurations at points (b) and (c).

well with experimental results for pure InAs films. ${ }^{4,8}$ These results show that a difference of only $11 \mathrm{meV}$ between the $R$ and $Z$ models allows for easy disordering of surface dimers at finite temperatures, explaining why the alternating dimer configuration of the $\alpha 2(2 \times 4)$ is not typically reported for binary systems.

The small difference in energy between the $R$ and $Z$ models from the GaAs to InAs lattice parameters shows that lattice mismatch strain has little influence on surface dimer placement in the $\alpha 2(2 \times 4)$ reconstruction. In order to examine the role of alloying, cations must be placed into the reconstruction unit cell in a way that mimics reality. It is well known that In surface segregates in InGaAs films. ${ }^{14}$ DFT calculations on the $\alpha 2(2 \times 4)$ reconstruction $(R$ model surface) and work by others on the $\beta 2(2 \times 4)$ surface $^{15}$ both show substantial $(>200 \mathrm{meV})$ energy increases as In is moved from surface positions to "bulklike" positions in the slab. The energy increase as In is moved deeper into the GaAs slab demonstrates a strong thermodynamic driving force for In surface segregation. Also, it is predicted that surface cation sites will have the most influence on surface dimer ordering. For these reasons, alloying below the surface cation positions will be ignored. The fraction of the 6 surface cation positions of a $(2 \times 4)$ GaAs slab occupied by In atoms is denoted $X_{\mathrm{In}}$.

DFT calculations of different In atom configurations in the surface sites of the $R$ and $Z$ models show that the placement of In relative to As dimers has a large effect on surface energy. The lowest energy In positions are those furthest from the As dimer, as they appear in Figs. 2(b) and 2(c). This means that the In preferentially sits in the cation-cation bond, which is oriented along the [110] [circled in Fig. 1(c)]. This cation-cation bond is generally in tension because the atom positions are constrained by the subsurface layers. Replacing $\mathrm{Ga}$ with the larger In reduces the bond tension, allowing for partial relaxation along the [110]. For a slab with surface composition $X_{\text {In }}=0.33$, the calculated length of a $\mathrm{Ga}-\mathrm{Ga}$ bond is $2.48 \AA$, while an In-Ga bond is $2.64 \AA$. DFT calcu-

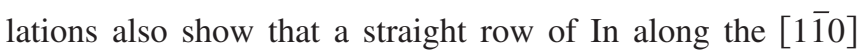
direction, as is the case in Fig. 2(b), has a much higher energy than an alternating configuration, displayed in Fig. 2(c), due to the inability of the In to relax in the $[1 \overline{1} 0]$. If the configuration in Fig. 2(b) is taken as a reference state, alter- 


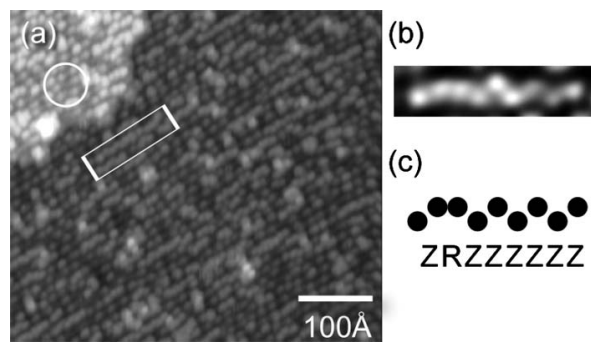

FIG. 3. (a) STM image of $h=25 \mathrm{ML} \operatorname{In}_{0.27} \mathrm{Ga}_{0.27} \mathrm{As}$ with no anneal taken at $I=100 \mathrm{pA}$ and $V=-3.13 \mathrm{~V}$. A circle outlines an area of $(4 \times 3)$, and a rectangle outlines one of $(2 \times 4)$. (b) Single dimer chain of $h=25 \mathrm{ML}$ $\mathrm{In}_{0.27} \mathrm{Ga}_{0.27} \mathrm{As}$ with $20 \mathrm{~min}$ annealing taken at $I=100 \mathrm{pA}$ and $V=-2.98 \mathrm{~V}$. (c) Schematic of dimer chain (b).

nating the In reduces the energy by $40 \mathrm{meV}$. However, alternating both the In and the As dimers, as is seen in Fig. 2(c), reduces the energy by $132 \mathrm{meV}$. Thus, the arrangement of In into a lower energy alternating configuration coupled with the preference of the In to sit in the cation-cation bond position opposite the As surface dimer induces the surface dimer to adopt an alternating configuration.

In may be arranged into numerous configurations with the same composition, $X_{\text {In }}$. Figure 2 shows the formation energy of the $R$ and $Z$ models with various In configurations as a function of composition (where formation energies are determined relative to a $Z$ model with $X_{\mathrm{In}}=0$ and $X_{\mathrm{In}}=1$ ). Configurations were determined by systematically placing In into the surface sites, determining the lowest energy configuration for that composition, and repeating this cycle until $X_{\text {In }}=1$ was reached. Figure 2 clearly shows that the $Z$ model has a lower formation energy than the $R$ model, and thus a lower surface energy, for all intermediate values of $X_{\mathrm{In}}$. The energy difference is small, $3 \mathrm{meV}$, for $X_{\mathrm{In}}=0$, increasing to a maximum of $92 \mathrm{meV}$ at $X_{\mathrm{In}}=0.33$, then decreasing with increasing $X_{\text {In }}$ until $X_{\text {In }}=1$ where the $R$ model is stabilized by $4 \mathrm{meV}$. The lowest energy configurations of the $Z$ and $R$ models at $X_{\text {In }}=0.33$ show that alternating both the In and the As dimers is critical to minimizing the energy of the system. Furthermore, the large energy difference across the composition range $0.167 \leqslant X_{\text {In }} \leqslant 0.667$ is significantly larger than the $11 \mathrm{meV}$ difference found for pure InAs, thereby making the $Z$ model more stable against thermal excitations that lead to a disordering of the dimers.

These results show a strong propensity for In alloyed GaAs $\alpha 2(2 \times 4)$ surfaces to exhibit the alternating dimer configuration of the $\alpha 2(2 \times 4)$ reconstruction. Figure 3(a) shows a scanning tunneling microscopy image of a $25 \mathrm{ML}$ $\mathrm{In}_{0.27} \mathrm{Ga}_{0.83} \mathrm{As} / \mathrm{GaAs}$ film grown at $480{ }^{\circ} \mathrm{C}$. It exhibits a mixed reconstruction with small domains of $\alpha 2(2 \times 4)$ reconstruction embedded in a $(4 \times 3)$ reconstruction matrix. ${ }^{16}$ Under these growth conditions, experimental and kinetic models predict a surface composition of $0.5 \leqslant X_{\text {In }} \leqslant 0.8{ }^{16}$ complicating direct comparison of this experiment with the DFT calculations. Further complications include the fact that the In may preferentially segregate to different reconstructions, and that the observed $\alpha 2(2 \times 4)$ domains are small, being an average of five dimers long. Nevertheless, the experimentally observed alloyed $\alpha 2(2 \times 4)$ chains exhibit a regular zigzag or alternating dimer configuration as can be seen in Figs. 3(b) and 3(c), consistent with the DFT results presented above. Statistical analysis of over 900 dimers in over 200 dimer chains for $25 \mathrm{ML}$ thick $\mathrm{In}_{0.27} \mathrm{Ga}_{0.27} \mathrm{As}$ films annealed for various times shows the incidence of alternating dimers for these films is $80 \%$, indicating that the alternating configuration is preferred under these conditions. The growth of thin films of InAs on GaAs by Patella et al. also results in a regular surface dimer alternation. ${ }^{17}$

We have examined the effect of both global lattice mismatch strain and local atomic size mismatch strain on the energy of two dimer configurations in the $\alpha 2(2 \times 4)$ reconstruction. We have demonstrated that for this particular alloy, local atomic size mismatch strain stabilizes the alternating dimer configuration. Alloyed films of $\operatorname{In}_{0.27} \mathrm{Ga}_{0.73} \mathrm{As} / \mathrm{GaAs}$ and InAs/GaAs exhibit a regularly alternating surface dimer in the $\alpha 2(2 \times 4)$ reconstruction, supporting these findings. Thus, we show that locally induced surface strain resulting from alloying has a large impact on the resulting surface reconstruction, which may in turn have a significant impact on the development of bulk ordering.

The authors wish to thank Chris Pearson, Alex Riposan for STM data, and Ernesto Placidi for InAs/GaAs data. We gratefully acknowledge support from DOE/BES (ER 46172). Sandia is a multiprogram laboratory operated by Sandia Corporation, a Lockheed Martin Company, for the United States Department of Energy under Contract No. DE-AC0494AL85000.

${ }^{1}$ P. Kratzer, E. Penev, and M. Scheffler, Appl. Surf. Sci. 216, 436 (2003). ${ }^{2}$ Z. X. Xie, X. Xu, J. Tang, and B. W. Mao, J. Phys. Chem. B 104, 11719 (2000).

${ }^{3}$ V. Bresslerhill, M. Wassermeier, K. Pond, R. Maboudian, G. A. D. Briggs, P. M. Petroff, and W. H. Weinberg, J. Vac. Sci. Technol. B 10, 1992 (1992).

${ }^{4}$ W. Barvosa-Carter, R. S. Ross, C. Ratsch, F. Grosse, J. H. G. Owen, and J. J. Zinck, Surf. Sci. 499, L129 (2002).

${ }^{5}$ S. B. Zhang and A. Zunger, Phys. Rev. B 53, 1343 (1996).

${ }^{6}$ C. Ratsch, Phys. Rev. B 63, 161306 (2001).

${ }^{7}$ A. Antons, Y. Cao, B. Voigtländer, K. Schroeder, R. Berger, and S. Blügel, Europhys. Lett. 62, 547 (2003).

${ }^{8}$ H. Yamaguchi, R. S. Ross, and Y. Horikoshi, Phys. Rev. B 51, 9836 (1995).

${ }^{9}$ G. R. Bell, M. Itoh, T. S. Jones, B. A. Joyce, and D. D. Vvendensky, Surf. Sci. 433, 455 (1999).

${ }^{10}$ G. Kresse and J. Hafner, Phys. Rev. B 47, 558 (1993); G. Kresse and J. Furthmüller, ibid. 54, 11169 (1996); G. Kresse and J. Hafner, J. Phys.: Condens. Matter 6, 8245 (1994).

${ }^{11}$ D. Vanderbilt, Phys. Rev. B 41, 7892 (1990).

${ }^{12}$ D. M. Ceperley and B. J. Alder, Phys. Rev. Lett. 45, 566 (1980).

${ }^{13}$ This lattice model, fit to DFT energies of seven dimer arrangements and performed on a $24 \times 24$ cell, includes interactions up to the third nearest neighbor and predicts a difference in energy between the $Z$ and $R$ models of $11 \mathrm{meV}$ per $(2 \times 4)$ surface unit cell.

${ }^{14}$ A. Bosacchi, F. Calonna, S. Franchi, P. Pascarella, P. Allegri, and V. Avanzini, J. Cryst. Growth 150, 185 (1995).

${ }^{15}$ J. H. Cho, S. B. Zhang, and A. Zunger, Phys. Rev. Lett. 84, 3654 (2000).

${ }^{16}$ A. Riposan, J. Mirecki Millunchick, and C. Pearson, J. Vac. Sci. Technol. A 24, 2041 (2006).

${ }^{17}$ F. Patella, A. Sgarlata, F. Arciprete, S. Nufris, P. D. Szkutnik, E. Placidi, M. Fanfoni, N. Motta, and A. Balzarotti, J. Phys.: Condens. Matter 16, S1503 (2004). 